\title{
Transforming Growth Factor Beta 1 Regulates Fibroblast Growth Factor 7 Expression in Schwann Cells
}

\author{
Doan Nguyen, PhD, ${ }^{1}$ Olawale A. R. Sulaiman, MD, PhD, FRCS ${ }^{2,3}$ \\ ${ }^{1}$ Department of Research, Ochsner Clinic Foundation, New Orleans, LA ${ }^{2}$ Department of Neurosurgery, Ochsner Clinic Foundation, New \\ Orleans, LA ${ }^{3}$ The University of Queensland Faculty of Medicine, Ochsner Clinical School, New Orleans, LA
}

\begin{abstract}
Background: Our previous work demonstrated that application of transforming growth factor beta 1 (TGF- $\beta 1$ ) and forskolin to the repair site after chronic denervation and axotomy has a mitogenic effect, reactivates Schwann cells (SCs), and supports axonal regeneration. We found decreased expression of fibroblast growth factor 7 (FGF-7), a factor involved in synaptic organization and maintenance. Using an in vitro system, we examined the molecular mechanism of TGF- $\beta 1$ and forskolin on the regulation of FGF-7 expression in SCs.

Methods: SCs were prepared from the sciatic nerve and stimulated with forskolin $(0.5 \mu \mathrm{M}), \mathrm{TGF}-\beta 1$ ( $1 \mathrm{ng} / \mathrm{mL})$, or TGF- $\beta 1+$ forskolin for 6 or 24 hours. SCs were also pretreated with LY2109761 $(0.5 \mu \mathrm{M})$, a TGF- $\beta$ receptor inhibitor, prior to stimulation with TGF$\beta 1+$ forskolin for 6 hours. Real-time TaqMan quantitative polymerase chain reaction analyses for FGF-7, myelin basic protein, and peripheral myelin protein 22 expression were performed. Cycle threshold $(\mathrm{Ct})$ data were normalized to a reference gene, and fold changes relative to untreated SCs were determined using the $2^{-\Delta \Delta \mathrm{Ct}}$ method. Statistical analysis was done using $t$ test $(P<0.05)$.

Results: TGF- $\beta 1$ alone or in combination with forskolin for 24 hours resulted in a 3.3- and 2.8-fold decrease in FGF-7 expression in SCs, respectively. No change in FGF-7 expression was found with forskolin alone. TGF- $\beta 1+$ forskolin treatment for 6 hours resulted in a 4.0-fold decrease in FGF-7 expression, while the addition of LY2109761 resulted in a 2.7-fold decrease in FGF-7 expression.

Conclusion: We showed that SC expression of FGF-7 is regulated by TGF- $\beta 1$. The positive effect of TGF- $\beta 1$ and forskolin on SC reactivation and axonal regeneration may involve modulation of FGF-7 expression and activity in SCs.
\end{abstract}

Keywords: Fibroblast growth factor 7, nerve injury, nerve regeneration, Schwann cells, transforming growth factor beta 1

Address correspondence to Olawale A. R. Sulaiman, MD, PhD, FRCS, Department of Neurosurgery, Ochsner Clinic Foundation, 1514 Jefferson Hwy., New Orleans, LA 70121. Tel: (504) 842-2128. Email: wsulaiman@ochsner.org

\section{INTRODUCTION}

Although peripheral nerves retain the capacity to regenerate, injuries that involve large nerve trunks such as the sciatic plexus and brachial plexus often result in poor and suboptimal functional recovery. ${ }^{1,2}$ The reason for the lack of functional recovery resides in the lack of sufficient mature axons reaching their targets, a failure attributable to loss of the growth-supportive environment provided by Schwann cells (SCs) in the distal stump of injured nerves. ${ }^{3-5}$

SCs play a major role during the degenerative process termed Wallerian degeneration and are intimately involved in subsequent regenerative processes. Loss of axonal contact triggers SC transition from a myelinating cell type to a nonmyelinating proliferative cell type. Expression of myelinassociated genes or proteins is downregulated. SCs proliferate in the distal nerve stump to establish cellular channels (bands of Büngner) through which regenerating axons traverse in the distal nerve stump to get to the denervated muscle targets. ${ }^{6}$ Expression of regeneration-associated genes such as neurotrophins and their receptors ${ }^{7}$; adhesion molecules ${ }^{8}$; and cytokines such as transforming growth factor beta 1 (TGF- $\beta 1)^{9}$ is upregulated in SCs to support the growth of axons through the distal nerve stump. TGF$\beta 1$ mediates the recruitment of macrophages into the denervated distal nerve stump, as well as protein mediators that suppress fibroblast proliferation. ${ }^{10,11}$ However, axonal growth is a slow and turbulent process because axons take many months or even years to reach their targets. The prolonged process leads to a condition of chronic denervation that alters the growth-supportive environment in the distal nerve stump.

Axon outgrowth can be stimulated both in vivo and in vitro by increasing intracellular cyclic adenosine monophosphate (cAMP) levels using forskolin or by blocking phosphodiesterases with rolipram. ${ }^{12-15}$ In vitro experiments established that $1 \mathrm{ng} / \mathrm{mL}$ TGF- $\beta 1$ and $0.5 \mu \mathrm{M}$ forskolin 
were optimal for maximal in vitro stimulation of SC proliferation. ${ }^{16,17}$ Ex vivo experiments demonstrated the benefit of combined TGF- $\beta 1$ and forskolin on the regenerative effect in chronically denervated nerves as old as 6 months. ${ }^{17-19}$ The mechanism underlying the regenerative effect of TGF- $\beta 1$ and forskolin on chronically denervated SCs is unknown.

Our previous work using a chronic denervation and axotomy rat model demonstrated that TGF- $\beta 1$ alone and with forskolin reversed the deleterious effect of chronic SC denervation and reactivated SCs to support axonal regeneration. ${ }^{17,19,20}$ Previous gene expression profiling identified fibroblast growth factor 7 (FGF-7), also known as keratinocyte growth factor, in the distal nerve stump. ${ }^{21}$ FGF-7 has been identified as a secreted factor involved in cell proliferation and tissue repair, as well as in synaptogenesis. ${ }^{21-23}$ The significance of FGF-7 and whether FGF-7 is expressed in SCs is not known. We found that TGF- $\beta 1$ and forskolin treatment at the site of injury and repair resulted in a 3-fold decrease in FGF-7 expression at the site of repair. This result suggests that the positive effect of TGF- $\beta 1$ on SC reactivation and axonal regeneration may involve downregulation of FGF-7 expression at the site of repair and distal to the site of repair.

In this study, we used quantitative polymerase chain reaction (qPCR) to confirm the microarray results for FGF-7 expression at the site of repair and in the distal nerve stump. Using an in vitro system, we examined the molecular mechanism of TGF- $\beta 1$ and forskolin regulation of FGF-7 expression in primary SCs.

\section{METHODS \\ Chronic Injury and Delayed Repair Model}

We used our rat model of tibial (TIB) nerve injury and delayed repair as previously described. ${ }^{16,17}$ All procedures were carried out on Sprague-Dawley (SD) rats and were approved and monitored by the Ochsner Institutional Animal Care and Use Committee. The first surgery involved incision of the rat hindlimb to expose the sciatic nerve, its TIB nerve, and common peroneal branches. The TIB nerve was transected, and its proximal stump was reflected backward and sutured into innervated muscle to prevent regeneration. The second surgery was performed after 8 weeks. The injured TIB nerve was reexposed, and end-to-end repair was done. After microsurgical repair, a $10 \mathrm{~mm}$ square piece of Surgifoam (Ethicon) presoaked with saline, forskolin $(0.5 \mu \mathrm{M})$, TGF- $\beta 1$ ( $1 \mathrm{ng} / \mathrm{mL})$, or TGF- $\beta 1$ + forskolin was applied by wrapping the Surgifoam around the TIB nerve repair site. The animals were allowed to recover, and regeneration of TIB nerves was allowed for 6 weeks. Nerve sections were taken from the proximal stump, the suture site region, and the distal nerve stump and were immediately frozen on dry ice for gene expression analysis.

\section{Schwann Cell Culture}

Rat SCs were isolated from the contralateral sciatic nerve of adult female SD rats, modified from an established protocol without the use of 2-deoxyuridine to inhibit fibroblast proliferation and a flame-narrowed borosilicate Pasteur pipette to triturate the tissues after enzymatic digestion. ${ }^{24}$ The epineurium sheath of the sciatic nerve was removed using fine forceps. The stripped nerve was cut into $1 \mathrm{~mm}$ segments, and 10-15 pieces were placed on the central portion of a $35 \mathrm{~mm}$ tissue dish containing $750 \mathrm{uL}$ of DMEM $/ 10 \%$ fetal bovine serum (FBS) (Atlanta Biologicals, Inc.) plus penicillin-streptomycin (PS). After 1 week, fibroblast outgrowth reached a near confluent monolayer around the nerve explants, and the nerve explants were removed and transferred to a new $35 \mathrm{~mm}$ tissue dish. By the fourth explants transfer, cellular outgrowth consisted principally of SCs, and explants were enzymatically dissociated overnight using Dispase II (0.25\%, Sigma Aldrich) and collagenase $(0.05 \%$, Worthington). The dissociated SCs were plated onto a poly-L-lysine-coated $60 \mathrm{~mm}$ tissue dish and maintained in complete DMEM/10\% FBS, PS, pituitary extract $(20 \mathrm{ug} / \mathrm{mL}$ Thermo Fisher Scientific), heregulin (10 ng/mL, BioLegend), forskolin (2 $\mu \mathrm{M}$, Sigma-Aldrich), and ascorbic acid (50 ug/mL, Sigma-Aldrich). SCs were subcultured and used between passages 3-6. SCs $(5 \times$ $10^{5}$ cells) were maintained on poly-L-lysine-coated tissue clusters for 6 days, starved for 17 hours, and stimulated with forskolin $(0.5 \mu \mathrm{M})$, TGF- $\beta 1$ ( $1 \mathrm{ng} / \mathrm{mL}$, R\&D Systems), or TGF- $\beta 1+$ forskolin for 24 hours. SCs were also treated with LY2109761 $(0.5 \mu \mathrm{M})$, a TGF- $\beta$ receptor type I/II (T $\beta$ RI/II) dual inhibitor (Selleckchem), for 15 minutes prior to stimulation with TGF- $\beta 1$ and forskolin for 6 hours or 24 hours.

\section{Real-Time Polymerase Chain Reaction}

Total RNA was extracted from SCs using the RNeasy Micro Kit per manufacturer's instruction (Qiagen) and quantified using a Nanodrop spectrophotometer (Thermo Fisher Scientific). Total RNA (100 ng) was used for complementary DNA (cDNA) synthesis using the iScript cDNA Synthesis Kit (Bio-Rad), and $10 \mathrm{ng}$ cDNA was used for real-time TaqMan qPCR (Thermo Fisher Scientific). Reactions were done in triplicate using the ABI 7500 thermal cycler (Applied Biosystems, Inc.). The cycle threshold (Ct) was determined using the default setting. The $\mathrm{Ct}$ of target genes was normalized to the ribosomal RPLS reference gene. Fold changes relative to untreated controls were analyzed using the $2^{-\Delta \Delta \mathrm{Ct}}$ method. ${ }^{25} \mathrm{~A} \geq 2$-fold change was used to represent differential gene expression. Statistical significance was analyzed using $t$ test $(P<0.05)$ and expressed as mean \pm standard deviation.

\section{Immunohistochemistry}

SCs $\left(5 \times 10^{5}\right.$ cells) were plated and maintained at $37^{\circ} \mathrm{C}$ for 24 hours. SCs were fixed in $4 \%$ paraformaldehyde at $4^{\circ} \mathrm{C}$ for 30 minutes and washed in phosphate buffered saline (PBS, Corning) 3 times for 5 minutes at room temperature (RT). SCs were blocked in 5\% normal goat serum PBS for 1 hour at RT, incubated with anti-FGF-7 (Santa Cruz Biotechnology) or anti-S100B (Proteintech) primary antibody at $4^{\circ} \mathrm{C}$ overnight and washed in PBS 3 times for 5 minutes at RT. The horseradish peroxidase (HRP) secondary antibody (Enzo Life Sciences) was added and incubated for $30 \mathrm{mi}-$ nutes, washed 3 times in PBS, and incubated for 30 minutes with avidin-biotin complex reagents (Vector Labs). SCs were washed in PBS and stained with 3, 3'-diaminobenzidine (DAB, Vector Labs), hematoxylin and eosin counterstained, and photographed on a Nikon E600 photomicroscope. The parallel control (without the primary antibody) was also analyzed. 


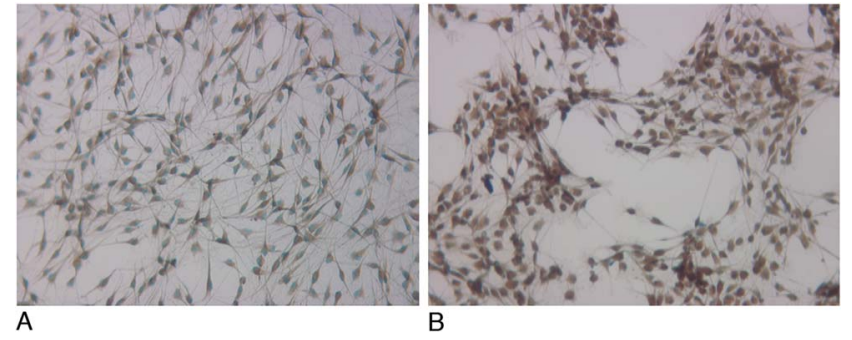

Figure 1. Immunocytochemical detection of (A) S100B and (B) fibroblast growth factor 7 (FGF-7) in primary Schwann cells (SCs) isolated from rat sciatic nerves. The positive staining indicates that these SCs are S100B-positive and express FGF-7 ( $x 10)$.

\section{RESULTS}

\section{TGF- $\beta 1$ Decreases FGF-7 Expression in the Chronically Denervated Nerve Stump}

Gene expression profiling of distal nerve stumps of chronically denervated SCs (8 weeks) that were repaired/treated with TGF- $\beta 1+$ forskolin or forskolin alone (6 weeks) showed that only TGF- $\beta 1+$ forskolin treatment resulted in a decreased expression of FGF-7. As stated in Methods, we used real-time TaqMan qPCR analysis to confirm the microarray results. We compared FGF-7 expression at the site of repair and distal nerve stump for TGF- $\beta 1+$ forskolin vs forskolin. Compared to forskolin treatment alone, TGF$\beta 1+$ forskolin treatment resulted in a 34.6-fold decrease in the expression of FGF-7 at the site of repair and a 24.2-fold decrease in the distal nerve stump.

\section{TGF- $\beta 1$ Decreases FGF-7 Expression in Schwann Cells}

Primary culture of rat SCs prepared from a normal sciatic nerve displayed a typical bipolar morphology and was immunopositive for S100B, a biomarker of SCs (Figure 1A). FGF-7 immunoreactivity was also positive in primary SCs (Figure 1B). To test the effect of TGF- $\beta 1$ on FGF-7 expression, SCs were cultured in the presence of TGF- $\beta 1(1 \mathrm{ng} / \mathrm{mL})$, forskolin $(0.5 \mu \mathrm{M})$, TGF- $\beta 1+$ forskolin, or media alone for 24 hours. As stated in Methods, the expression of FGF-7 in primary SCs was determined by real-time TaqMan qPCR, and fold change was analyzed relative to the untreated control. Expression of FGF-7 significantly decreased 3.3-fold with TGF- $\beta 1$ treatment and 2.8-fold with TGF- $\beta 1+$ forskolin $(P<0.05)$ after 24 hours (Figure 2). Forskolin treatment alone also decreased FGF-7 expression, but the decrease did not reach the 2-fold cutoff.

Expression of myelin basic protein (MBP) and peripheral myelin protein 22 (PMP-22), markers for SC differentiation, were also analyzed by real-time TaqMan qPCR. The expression of MBP decreased 3.3-fold with TGF- $\beta 1$ treatment and 5.8-fold with TGF- $\beta 1+$ forskolin. MBP expression showed a downward trend after forskolin-only treatment, but the expression was less than the 2-fold cutoff. Expression of PMP-22 was not different from the control after forskolin or TGF- $\beta 1$ treatment. However, PMP-22 expression showed a downward trend with TGF- $\beta 1+$ forskolin treatment relative to the control.

\section{FGF-7 Is Regulated by the TGF- $\beta$ Receptor} Signaling Pathway

Based on the observation that FGF-7 expression is decreased in the presence of TGF- $\beta 1$, we sought to determine if inhibition of the TGF- $\beta 1$ ligand mediated activation of the TGF- $\beta$ receptor signaling effect on FGF-7 expression. As stated in Methods, SCs were pretreated with LY2109761, a T $\beta R I / I I$ dual inhibitor, for 15 minutes followed by TGF- $\beta 1$ + forskolin stimulation for 6 hours. Expression of FGF-7 decreased 4-fold after a 6-hour incubation with TGF- $\beta 1+$ forskolin; however, in the presence of the LY2109761 dual inhibitor, the impact of TGF- $\beta 1+$ forskolin treatment on FGF7 expression was less, with a decrease of 2.7-fold relative to the control (Figure 3). FGF-7 expression levels after 6 hours with the dual inhibitor and TGF- $\beta 1+$ forskolin were similar to FGF-7 expression at 24 hours.

LY2109761 had no effect on MBP expression after TGF- $\beta 1$ + forskolin stimulation, whereas TGF- $\beta 1$ + forskolin treatment decreased PMP-22 expression similar to the effect observed at 24 hours, and PMP-22 expression showed an upward trend in the presence of LY2109761.

\section{DISCUSSION}

In previous work, we demonstrated the positive effect of TGF- $\beta 1$ and forskolin treatment on SC reactivation and axonal regeneration in the chronically denervated nerve stump. ${ }^{17,19,20}$ The decreased expression of FGF-7 two months after TGF- $\beta 1$ and forskolin treatment of a chronically denervated nerve stump suggests negative regulation of FGF-7 by TGF- $\beta 1$, and forskolin may be needed to promote or maintain axonal regeneration. To our knowledge, ours is the first report of FGF-7 expression in the TIB nerve and SCs.

In addition, we showed that TGF- $\beta 1$ and forskolin decreased MBP and PMP-22 expression, suggesting that TGF- $\beta 1$ and forskolin treatment maintain SCs in a nonmyelinating proliferative state in vivo. TGF- $\beta 1+$ forskolin decreased MBP expression at 24 hours but did not decrease

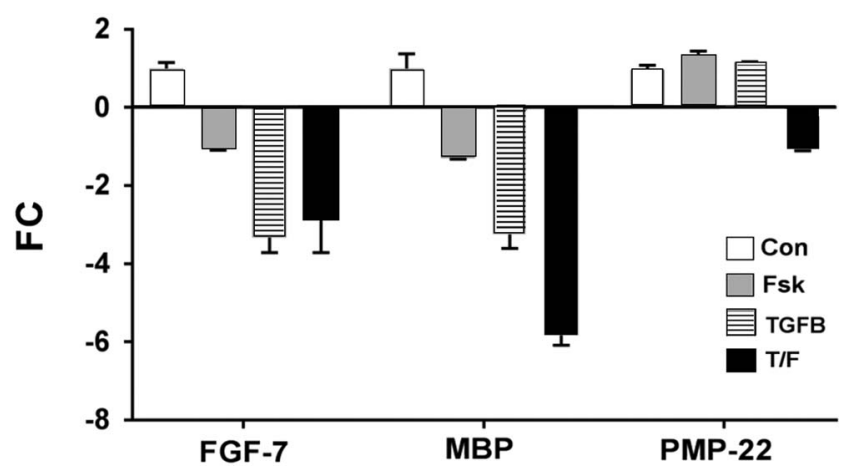

Figure 2. Fold changes (FC) in expression of fibroblast growth factor 7 (FGF-7), myelin basic protein (MBP), and peripheral myelin protein 22 (PMP-22) relative to untreated controls (Con) after 24-hour treatment with forskolin (Fsk), transforming growth factor beta 1 (TGFB), or transforming growth factor beta $1+$ forskolin (T/F). Expression of FGF7 and MBP significantly decreased with TGFB and T/F treatments $(n=3, P<0.05)$. 


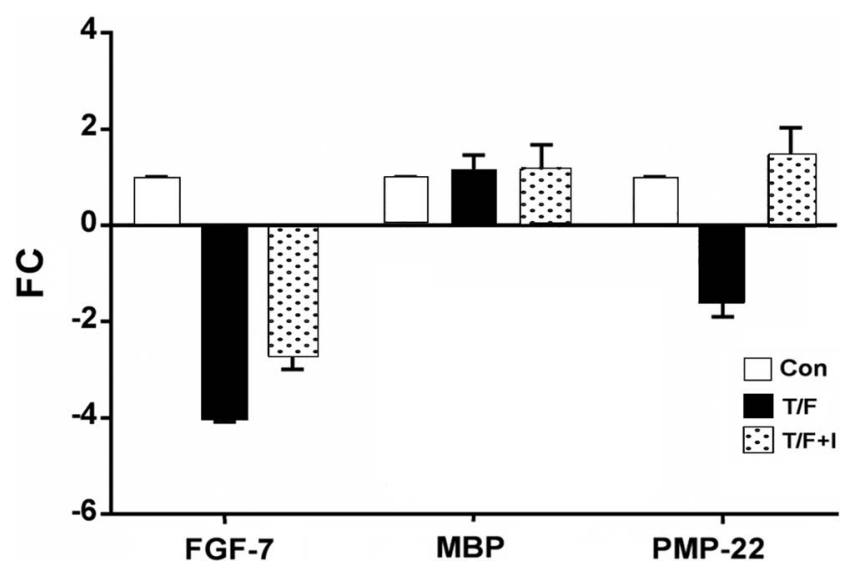

Figure 3. Fold changes (FC) in expression of fibroblast growth factor 7 (FGF-7), myelin basic protein (MBP), and peripheral myelin protein 22 (PMP-22) relative to untreated controls (Con). Schwann cells were stimulated with transforming growth factor beta 1 (TGF- $\beta 1$ ) + forskolin (T/F) alone or with T/F and LY2109761 (T/F+I), a TGF- $\beta$ receptor type I/II (T $\beta R 1 / I I)$ inhibitor, for 6 hours $(n=3)$. Blocking of T $\beta R 1 / I I$ decreased the effect of T/F treatment on FGF-7 expression $(P<0.05)$.

MBP expression at 6 hours. The difference may be that MBP, which is constitutively expressed by SCs in vitro, may require extended treatment longer than 24 hours to be affected by TGF- $\beta 1+$ forskolin treatment. The negative effect on FGF7 expression by TGF- $\beta 1$ appeared to be partially mediated by $T \beta R \mathrm{RI} / \mathrm{Il}$ activation of the TGF- $\beta 1-$ mediated signaling pathway, as FGF-7 expression was not completely suppressed. TGF- $\beta 1$ and forskolin, which activate different signaling pathways, may act in an antagonistic way in regulating FGF-7 expression as shown with T $\beta R \mathrm{RI} / \mathrm{II}$ inhibition in SCs, and suppression of FGF-7 may be an important aspect of chronic neural repair and regeneration.

The effects of TGF- $\beta$ on SCs are complex, and outcomes are dependent on the metabolic state as well as the presence or absence of other mitogenic factors. ${ }^{12,20,26}$ TGF- $\beta$ acts as a mitogen in SC culture, ${ }^{16}$ in the normal milieu of the nerve and activated macrophages, ${ }^{10,11}$ and in neurons. ${ }^{27,28}$ Furthermore, SCs also synthesize and secrete TGF- $\beta$. The effects of TGF- $\beta$ on SCs in the presence of neurons are more of a modulatory role and are in part responsible for the switch from a quiescent myelinating phenotype to a more active proliferative phenotype in the absence of neurons ${ }^{28}$ as happens during Wallerian degeneration. ${ }^{29}$

The positive effect of TGF- $\beta 1$ and forskolin on SC reactivation and axonal regeneration may involve the modulation of FGF-7 expression. FGF-7 is a member of the FGF intercellular signaling molecule involved in a wide range of biologic processes, including cell proliferation, migration, tissue repair, and response to injury. ${ }^{21}$ FGF-7 was first identified as a protein secreted from mesenchymal cells to repair epithelial tissues. FGF-7, FGF-10, and FGF-22 are closely related members of the FGF family. These factors have been identified as target-derived factors and as synaptic organizers involved in the formation and maintenance of nerve terminals at synaptic sites. ${ }^{22,23}$ During neuromuscular junction development, TGF- $\beta 1$ mediates synaptic formation similar to other astrocyte-derived synaptogenic factors and leads to doubling of acetylcholine receptor clusters at the nerve-muscle contact. ${ }^{30}$ In vitro studies using SC-conditioned media found the positive effect on synaptogenesis at the neuromuscular junction and showed that TGF- $\beta 1$ acts as SC-derived synaptogenic factors. 9,30 In a study published in 2012, FGF-7 was shown to function as an inhibitory regulator of synaptogenesis, as FGF-7 knockout mice are prone to epileptogenesis. ${ }^{31}$ Glial cells, including SCs, not only serve as support cells but are also active participants in modulating presynaptic nerve terminals and postsynaptic specialization. ${ }^{30,32}$ FGF-7 has also been identified in small-diameter neurons of the dorsal root ganglion and localized in large dense-core vesicles in nociceptive neurons. ${ }^{33}$ FGF-7 is postulated to exert an excitatory postsynaptic response as FGF-7-deficient mice have reduced acute and inflammatory nociceptive responses. ${ }^{33}$

The molecular mechanism underlying TGF- $\beta 1$ modulation of FGF-7 expression is not known. Our results are consistent with prior in vitro work in which the combination of TGF- $\beta 1$ + forskolin added to cultures of chronically denervated isolated SCs for 48 hours was sufficient to increase axon survival when used in vivo as a nerve bridge. ${ }^{14}$ Our results are also consistent with the hypothesis that while forskolin is sufficient to activate cAMP functions in $\mathrm{SCs}^{34}$ and hence mimic the action of various growth factors such as glial cell-derived neurotrophic factor, ${ }^{35}$ the effect of TGF- $\beta 1$ and its modulatory action on other growth factors are important for the full effect to be seen. ${ }^{27,35-37}$

As such, understanding the role of FGF-7 in axonal repair and regeneration could provide the basis for development of alternative approach to modulate $\mathrm{SC}$ response and synaptic organization.

\section{CONCLUSION}

We showed that SC expression of FGF-7 is regulated by TGF- $\beta 1$. The positive effect of TGF- $\beta 1$ and forskolin on SC reactivation and axonal regeneration may involve modulation of FGF-7 expression and activity in SCs.

\section{ACKNOWLEDGMENTS}

The authors have no financial or proprietary interest in the subject matter of this article. A portion of this work was presented at the Society for Neuroscience Annual Meeting (October 2015, Chicago, IL) and at the Louisiana Neurosurgical Society (January 2016, New Orleans, LA).

\section{REFERENCES}

1. Fu SY, Gordon T. Contributing factors to poor functional recovery after delayed nerve repair: prolonged axotomy. $J$ Neurosci. 1995 May;15(5 Pt 2):3876-3885.

2. Sulaiman W, Gordon T. Neurobiology of peripheral nerve injury, regeneration, and functional recovery: from bench top research to bedside application. Ochsner J. 2013 Spring;13(1):100-108.

3. Gordon T. The biology, limits, and promotion of peripheral nerve regeneration in rats and humans. In: Tubbs RS, Rizk E, Shoja MM, Loukas M, Barbaro N, Spinner RJ eds. Nerves and Nerve Injuries: Vol 2: Pain, Treatment, Injury, Disease, and Future Directions. New York, NY: Academic Press; 2015:993-1019. 
4. Gordon T, Sulaiman O, Boyd JG. Experimental strategies to promote functional recovery after peripheral nerve injuries. $J$ Peripher Nerv Syst. 2003 Dec;8(4):236-250.

5. Sulaiman O, Boyd J, Gordon T. 36. Axonal regeneration in the peripheral nervous system of mammals. In: Kettermann $\mathrm{H}$, Ransom B, eds. Neuroglia. Oxford, UK: Oxford University Press; 2005:454-466.

6. Li H, Terenghi G, Hall SM. Effects of delayed re-innervation on the expression of c-erbB receptors by chronically denervated rat Schwann cells in vivo. Glia. 1997 Aug;20(4):333-347.

7. Tomita K, Kubo T, Matsuda K, et al. The neurotrophin receptor p75NTR in Schwann cells is implicated in remyelination and motor recovery after peripheral nerve injury. Glia. 2007 Aug 15;55(11):1199-1208.

8. Avari P, Huang W, Averill S, et al. The spatiotemporal localization of JAM-C following sciatic nerve crush in adult rats. Brain Behav. 2012 Jul;2(4):402-414. doi: 10.1002/brb3.63.

9. Feng Z, Ko CP. Schwann cells promote synaptogenesis at the neuromuscular junction via transforming growth factor-beta 1 . J Neurosci. 2008 Sep 24;28(39):9599-9609. doi: 10.1523/JNEUROSCI.2589-08.2008.

10. Assoian RK, Fleurdelys BE, Stevenson HC, et al. Expression and secretion of type beta transforming growth factor by activated human macrophages. Proc Natl Acad Sci U. S. A. 1987;84(17):6020-6024.

11. Einheber S, Hannocks MJ, Metz CN, Rifkin DB, Salzer JL. Transforming growth factor-beta 1 regulates axon/Schwann cell interactions. J Cell Bio. 1995 Apr;129(2):443-458.

12. Stewart HJ, Eccleston PA, Jessen KR, Mirsky R. Interaction between CAMP elevation, identified growth factors, and serum components in regulating Schwann cell growth. J Neurosci Res. 1991 Oct;30(2):346-352.

13. Aglah C, Gordon T, Posse de Chaves El. CAMP promotes neurite outgrowth and extension through protein kinase $A$ but independently of Erk activation in cultured rat motoneurons. Neuropharmacology. 2008 Jul;55(1):8-17. doi: 10.1016/j.neuropharm.2008.04.005.

14. Monje PV, Bartlett Bunge M, Wood PM. Cyclic AMP synergistically enhances neuregulin-dependent ERK and Akt activation and cell cycle progression in Schwann cells. Glia. 2006 Apr 15;53(6):649-659.

15. Udina E, Ladak A, Furey M, Brushart T, Tyreman N, Gorden T. Rolipram-induced elevation of CAMP or chondroitinase $A B C$ breakdown of inhibitory proteoglycans in the extracellular matrix promotes peripheral nerve regeneration. Exp Neurol. 2010 May;223(1):143-152. doi: 10.1016/j.expneurol.2009.08.026.

16. Ridley AJ, Davis JB, Stroobant P, Land H. Transforming growth factors-beta 1 and beta 2 are mitogens for rat Schwann cells. $J$ Cell Biol. 1989 Dec;109(6 Pt 2):3419-3424.

17. Sulaiman OA, Gordon T. Transforming growth factor-beta and forskolin attenuate the adverse effects of long-term Schwann cell denervation on peripheral nerve regeneration in vivo. Glia. 2002 Mar 1;37(3):206-218.

18. Sulaiman OA, Gordon T. Effects of short- and long-term Schwann cell denervation on peripheral nerve regeneration, myelination, and size. Glia. 2000 Dec;32(3):234-246.

19. Sulaiman W, Dreesen T. Effect of local application of transforming growth factor- $\beta$ at the nerve repair site following chronic axotomy and denervation on the expression of regeneration associated genes. J Neurosurg. 2014 Oct;121(4):859-874. doi: 10.3171/2014.4.JNS131251.

20. Sulaiman W, Dreesen T, Nguyen D. Single local application of TGF- $\beta$ promotes a proregenerative state throughout a chronically injured nerve. Neurosurgery. 2018 Jun 1;82(6):894-902. doi: 10.1093/neuros/nyx362.

21. Umermori H, Linhoff MW, Omitz DM, Sanes JR. FGF22 and its close relatives are presynaptic organizing molecules in the mammalian brain. Cell. 2004 Jul 23;118(2):257-270.

22. Fox MA, Sanes JR, Borza DB, et al. Distinct target-derived signals organize formation, maturation, and maintenance of motor nerve terminal. Cell. 2007 Apr 6;129(1):179-193. doi: 10.1016/j.cell.2007.02.035.

23. Terauchi $A$, Johnson-Venkatesh $E M$, Toth $A B$, Javed $D$, Sutton $M A$, Umemori H. Distinct FGFs promote differentiation of excitatory and inhibitory synapses. Nature. 2010 Jun 10;465(7299):783-787. doi: 10.1038/nature09041.

24. Casella GT, Wieser R, Bunge RP, et al. Density dependent regulation of human Schwann cell proliferation. Glia. 2000 Apr;30(2):165-177.

25. Livak KJ, Schmittgen TD. Analysis of relative gene expression data using real-time quantitative PCR and the $2^{-\Delta \Delta \mathrm{Ct}}$ method. Methods. 2001 Dec;25(4):402-408. doi: 10.1006/meth.2001.1262.

26. Dong Z, Dean C, Walters JE, Mirsky R, Jessen KR. Response of Schwann cells to mitogens in vitro is determined by pre-exposure to serum, time in vitro, and developmental age. Glia. 1997 Jul;20(3):219-230.

27. Schober A, Hertel R, Arumäe U, et al. Glial cell line-derived neurotrophic factor rescues target-deprived sympathetic spinal cord neurons but requires transforming growth factor-beta as cofactor in vivo. J Neurosci. 1999 Mar 15;19(6):2008-2015.

28. Guénard V, Rosenbaum T, Gwynn LA, Doetschman T, Ratner N, Wood PM. Effect of transforming growth factor-beta 1 and -beta 2 on Schwann cell proliferation on neurites. Glia. 1995 Apr;13(4):309-318.

29. Yang DP, Zhang DP, Mak KS, Bonder DE, Pomeroy SL, Kim HA. Schwann cell proliferation during Wallerian degeneration is not necessary for regeneration and remyelination of the peripheral nerves: axon-dependent removal of newly generated Schwann cells by apoptosis. Mol Cell Neurosci. 2008 May;38(1):80-88. doi: 10.1016/j.mcn.2008.01.017.

30. Feng Z, Ko CP. Neuronal glia interactions at the vertebrate neuromuscular junction. Curr Opin Pharmacol. 2007 Jun;7(3):316-324.

31. Lee $\mathrm{CH}$, Javed $\mathrm{D}$, Althaus AL, Parent JM, Umemori $\mathrm{H}$. Neurogenesis is enhanced and mossy fiber sprouting arises in FGF7-deficient mice during development. Mol Cell Neurosci. 2012 Nov;51(3-4):61-67. doi: 10.1016/j.mcn.2012.07.010.

32. Kang H, Tian L, Mikesh M, Lichtman JW, Thompson WJ. Terminal Schwann cells participate in neuromuscular synapse remodeling during reiinervation following nerve injury. $J$ Neurosci. 2014 Apr 30;34(18):6323-6333. doi: 10.1523/JNEUROSCI.4673-13.2014.

33. Liu H, Wu QF, Li JY, et al. Fibroblast growth factor 7 is a nociceptive modulator secreted via large dense-core vesicles. J Mol Cell Biol. 2015 Oct;7(5):466-475. doi: $10.1093 / \mathrm{jmcb} / \mathrm{mj} \mathbf{0 1 9}$.

34. lacovelli J, Lopera J, Bott M, et al. Serum and forskolin cooperate to promote $\mathrm{G} 1$ progression in Schwann cells by differentially regulating cyclin D1, cyclin E1, and p27Kip expression. Glia. 2007 Dec;55(16):1638-1647. doi: 10.1002/glia.20578.

35. Höke A, Gordon T, Zochodne DW, Sulaiman OA. A decline in glial cell-line-derived neurotrophic factor expression is associated with impaired regeneration after long-term Schwann cell denervation. Exp Neurol. 2002 Jan;173(1):77-85. doi: 10.1006/exnr.2001.7826. 
36. Morgan L, Jessen KR, Mirsky R. Negative regulation of the PO gene in Schwann cells: suppression of PO mRNA and protein induction in cultured Schwann cells by FGF2 and TGF beta 1, TGF beta 2 and TGF beta 3. Development. 1994 Jun;120(6):1399-1409.
37. Krieglstein $K$, Henheik $P$, Farkas $L$, et al. Glial cell line-derived neurotrophic factor requires transforming growth factor-beta for exerting its full neurotrophic potential on peripheral and CNS neurons. J Neurosci. 1998 Dec 1;18(23):9822-9834.

This article meets the Accreditation Council for Graduate Medical Education and the American Board of Medical Specialties Maintenance of Certification competencies for Patient Care, Medical Knowledge, and Systems-Based Practice. 